\title{
LOCAL EXTREMA IN RANDOM TREES
}

\author{
LANE CLARK
}

Received 23 March 2004 and in revised form 8 November 2005

The number of local maxima (resp., local minima) in a tree $T \in \mathscr{T}_{n}$ rooted at $r \in[n]$ is denoted by $M_{r}(T)$ (resp., by $m_{r}(T)$ ). We find exact formulas as rational functions of $n$ for the expectation and variance of $M_{1}(T)$ and $m_{n}(T)$ when $T \in \mathscr{T}_{n}$ is chosen randomly according to a uniform distribution. As a consequence, a.a.s. $M_{1}(T)$ and $m_{n}(T)$ belong to a relatively small interval when $T \in \mathscr{T}_{n}$.

\section{Introduction}

The extension of permutation statistics to labelled trees is the subject of a number of articles. Generating functions for the number of labelled trees of several types according to the number of ascents and descents are given in [4]. A functional equation satisfied by the generating function for the number of labelled trees according to the number of descents and leaves is given in [5]. Central and local limit theorems for the number of ascents or of descents in uniformly random labelled trees are given in [1]. A functional equation satisfied by the generating function for the number of labelled trees according to the number of inversions is given in [7]. Related results are contained in [6]. A formula for the expected number of inversions of a uniformly random labelled tree is given in [9]. Formulas for the expectation and variance of the number of inversions of a uniformly random labelled tree are given in [2].

Local extrema (in the literature as local maxima and local minima; peaks and troughs; collectively turning points; related to phases) in permutations have a long history; see [10] and references there in. The examination of local maxima (equivalently, local minima) in permutations is more recent. A recurrence relation and a generating function for the number of permutations according to the number of local maxima are given in [10]. A central limit theorem for the number of local maxima in a uniformly random permutation also is given in [10]. In this note, we extend local extrema in permutations to labelled trees and examine local maxima (equivalently, local minima) in uniformly random labelled trees.

For $n \geq 2$, let $\mathscr{T}_{n}$ denote the set of trees with vertex set $[n]:=\{1, \ldots, n\}$. When $T_{1}, T_{2} \in$ $\mathscr{T}_{n}, T_{1}=T_{2}$ if and only if $T_{1}$ and $T_{2}$ have the same edge set. Let $T \in \mathscr{T}_{n}, r \in[n]$, and 
Table 1.1

\begin{tabular}{l|c|rrrrrr}
\hline & $T_{1}(n, k)=t_{n}(n, k)$ & 0 & 1 & 2 & 3 & 4 & $\cdots$ \\
\hline \multirow{4}{*}{$n$} & 2 & 1 & 0 & 0 & 0 & 0 & $\cdots$ \\
& 3 & 2 & 1 & 0 & 0 & 0 & $\cdots$ \\
& 5 & 9 & 1 & 0 & 0 & $\cdots$ \\
& 5 & 24 & 73 & 27 & 1 & 0 & $\cdots$ \\
\hline
\end{tabular}

distinct $i, j, k \in[n]$. We say $T$ rooted at $r$ has a local maximum at path $i j k$ if and only if $j>i, k$ and the path in $T$ from $r$ to $k$ contains the path $i j k$. Similarly, $T$ rooted at $r$ has a local minimum at path $i j k$ if and only if $j<i, k$ and the path in $T$ from $r$ to $k$ contains the path $i j k$. Let $M_{r}(T)=M_{r, n}(T)$ (resp., $m_{r}(T)=m_{r, n}(T)$ ) denote the number of local maxima (resp., local minima) of $T \in \mathscr{T}_{n}$ rooted at $r$. Then $M_{r}(T), m_{r}(T) \in\{0, \ldots, n-2\}$. Let $T_{r}(n, k)$ (resp., $t_{r}(n, k)$ ) denote the number of trees in $\mathscr{T}_{n}$ rooted at $r$ with precisely $k$ local maxima (resp., $k$ local minima). Then $T_{r}(n, k)=t_{r}(n, k)=0$ for $k \notin\{0, \ldots, n-2\}$ and $\sum_{k=0}^{n-2} T_{r}(n, k)=\sum_{k=0}^{n-2} t_{r}(n, k)=n^{n-2}$. As with the other statistics extended to labelled trees, roots $r=1, n$ are appropriate. The values of $T_{1}(n, k)=t_{n}(n, k)$ (see Lemma 2.1) are given in Table 1.1 for $2 \leq n \leq 5$ and $0 \leq k \leq n-2$.

We work in the probability space $\Omega_{n}$ consisting of all trees in $\mathscr{T}_{n}$, where each tree is chosen randomly according to a uniform distribution. Hence, $\operatorname{Pr}(T)=1 / n^{n-2}$ for $T \in \mathscr{T}_{n}$. A property $Q$ of trees in $\left\{\mathscr{T}_{n}\right\}$ holds asymptotically almost surely (a.a.s.) on $\left\{\Omega_{n}\right\}$ if and only if $\lim _{n \rightarrow \infty} \operatorname{Pr}\left(T \in \mathscr{T}_{n}: T\right.$ has property $\left.Q\right) \rightarrow 1$ as $n \rightarrow \infty$.

The parameters $M_{r}$ and $m_{r}$ are then random variables on $\Omega_{n}$ whose exact expectations $E\left(M_{r}\right), E\left(m_{r}\right)$ and variances $\sigma^{2}\left(M_{r}\right), \sigma^{2}\left(m_{r}\right)$ we find as rational functions of $n(r=1, n)$. From Theorem 2.5,

$$
\begin{aligned}
E\left(M_{1}\right) & =E\left(m_{n}\right)=\frac{2 n^{3}-3 n^{2}-5 n+6}{6 n^{2}} \\
\sigma^{2}\left(M_{1}\right) & =\sigma^{2}\left(m_{n}\right)=\frac{7 n^{5}-20 n^{4}+75 n^{3}-40 n^{2}-322 n+300}{60 n^{4}} .
\end{aligned}
$$

As a consequence, a.a.s. on $\left\{\Omega_{n}\right\}$,

$$
E\left(M_{1}\right)-\omega(n) \sigma\left(M_{1}\right)<M_{1}, m_{n}<E\left(M_{1}\right)+\omega(n) \sigma\left(M_{1}\right)
$$

where $\omega(n) \rightarrow \infty$ arbitrarily slowly as $n \rightarrow \infty$. (See Corollary 2.6 for this and further results.)

We mention that should $\left(M_{1}-E\left(M_{1}\right)\right) / \sigma\left(M_{1}\right) \stackrel{d}{\rightarrow} N(0,1)$, we could only conclude the above inequality for $M_{1}, m_{n}$ a.a.s. on $\left\{\Omega_{n}\right\}$. Of course, asymptotic normality of $M_{1}, m_{n}$ gives more information about the distribution of $M_{1}, m_{n}$ than their a.a.s. properties.

Let $\mathbb{N}$ denote the nonnegative integers and let $\mathbb{R}$ denote the real numbers. The expectation of a random variable $X$ is denoted by $E(X)$ and its variance by $\operatorname{Var}(X)$. We refer the reader to Moon [8] for trees and Durrett [3] for probability. 


\section{Results}

We first show that local maxima in trees rooted at $r$ and local minima in trees rooted at $n+1-r$ are equidistributed.

LEMma 2.1. For $r \in[n]$,

$$
T_{r}(n, k)=t_{n+1-r}(n, k) \quad(0 \leq k \leq n-2) .
$$

Proof. The bijection $i \mapsto n+1-i(i \in[n])$ induces a bijection $T \in \mathscr{T}_{n} \mapsto T^{\prime} \in \mathscr{T}_{n}$, where $T \simeq T^{\prime}$. Then $r, \ldots, i, j, k$ (with $j>i, k$ ) is a path in $T$ if and only if $n+1-r, \ldots, n+1-$ $i, n+1-j, n+1-k$ (with $n+1-j<n+1-i, n+1-k$ ) is a path in $T^{\prime}$. Hence, $M_{r}(T)=$ $m_{n+1-r}\left(T^{\prime}\right)$. Consequently, $T_{r}(n, k)=t_{n+1-r}(n, k)$ for $0 \leq k \leq n-2$.

In view of Lemma 2.1 , we consider only $M_{1}$.

Let $(x)_{0}=x^{0}=1(x \in \mathbb{R})$ and $(x)_{k}=(x) \cdots(x-k+1)(k \geq 1, x \in \mathbb{R})$. For $n \in \mathbb{N}$ and $a, x \in \mathbb{R}$, let

$$
\begin{aligned}
& E_{n}(x)=\sum_{k=0}^{n} \frac{x^{k}}{k !}, P_{n}(x)=\sum_{k=0}^{n}(n)_{k} x^{k}, \quad Q_{n, a}(x)=\sum_{k=0}^{n}(n)_{k}(k+a) x^{k}, \\
& R_{n}(x)=\sum_{k=0}^{n}(n)_{k}(k+1)(k+7) x^{k} .
\end{aligned}
$$

We require the following technical result which allows us to calculate the exact expectation and variance of $M_{1}$ as rational functions of $n$.

Lemma 2.2. For $n, m-1 \in \mathbb{N}$,

$$
P_{n}\left(\frac{1}{m}\right)=\frac{n !}{m^{n}} E_{n}(m)
$$

for $n-1, m-1 \in \mathbb{N}$, and $a \in \mathbb{R}$,

$$
Q_{n, a}\left(\frac{1}{m}\right)=\frac{n !}{m^{n}}(n+a) E_{n}(m)-\frac{n !}{m^{n-1}} E_{n-1}(m),
$$

and for $n-2, m-1 \in \mathbb{N}$,

$$
R_{n}\left(\frac{1}{m}\right)=\frac{n !}{m^{n}}\left(n^{2}+8 n+7\right) E_{n}(m)-\frac{n !}{m^{n-1}}(2 n+7) E_{n-1}(m)+\frac{n !}{m^{n-2}} E_{n-2}(m) .
$$

Proof. (All derivatives are with respect to real $x$ ). First,

$$
\frac{x^{n} P_{n}\left(x^{-1}\right)}{n !}=\sum_{k=0}^{n} \frac{x^{n-k}}{(n-k) !}=E_{n}(x)
$$

so that

$$
P_{n}(x)=n ! x^{n} E_{n}\left(x^{-1}\right)
$$


3870 Local extrema in random trees

and hence

$$
P_{n}\left(\frac{1}{m}\right)=\frac{n !}{m^{n}} E_{n}(m)
$$

Next, (2.7) gives

$$
\begin{aligned}
Q_{n, a}(x) & =x P_{n}^{\prime}(x)+a P_{n}(x) \\
& =n ! x^{n}(n+a) E_{n}\left(x^{-1}\right)-n ! x^{n-1} E_{n-1}\left(x^{-1}\right),
\end{aligned}
$$

and hence

$$
Q_{n, a}\left(\frac{1}{m}\right)=\frac{n !}{m^{n}}(n+a) E_{n}(m)-\frac{n !}{m^{n-1}} E_{n-1}(m) .
$$

Finally, (2.7) gives

$$
\begin{aligned}
R_{n}(x)= & x^{2} P_{n}^{\prime \prime}(x)+9 x P_{n}^{\prime}(x)+7 P_{n}(x) \\
= & n ! x^{n}\left(n^{2}+8 n+7\right) E_{n}\left(x^{-1}\right)-n ! x^{n-1}(2 n+7) E_{n-1}\left(x^{-1}\right) \\
& +n ! x^{n-2} E_{n-2}\left(x^{-1}\right),
\end{aligned}
$$

and hence

$$
R_{n}\left(\frac{1}{m}\right)=\frac{n !}{m^{n}}\left(n^{2}+8 n+7\right) E_{n}(m)-\frac{n !}{m^{n-1}}(2 n+7) E_{n-1}(m)+\frac{n !}{m^{n-2}} E_{n-2}(m) .
$$

Corollary 2.3. For $j, n-1 \in \mathbb{N}$ with $0 \leq j \leq n$,

$$
Q_{n-j, j}\left(\frac{1}{n}\right)=n \text {. }
$$

Proof. For $0 \leq j \leq n-1$, our result follows from Lemma 2.2. For $j=n$, our result follows from the definition of $Q_{n-j, j}(x)$.

We require the following result of Moon [8].

Theorem 2.4 (Moon [8]). Let $F$ be a forest with vertex set $[n]$ having $\omega$ components of orders $p_{1}, \ldots, p_{\omega}$. Then the number of distinct trees in $\mathscr{T}_{n}$ containing $F$ is $p n^{\omega-2}$, where $p=p_{1} \ldots p_{\omega}$.

We now give our main result. Here $M_{1}=M_{1, n}$.

Theorem 2.5. For $\Omega_{n}(n \geq 2)$,

$$
\begin{aligned}
& E\left(M_{1}\right)=\frac{2 n^{3}-3 n^{2}-5 n+6}{6 n^{2}}, \\
& E\left(M_{1}^{2}\right)=\frac{20 n^{6}-39 n^{5}-115 n^{4}+495 n^{3}-175 n^{2}-1266 n+1080}{180 n^{4}},
\end{aligned}
$$

hence,

$$
\sigma^{2}\left(M_{1}\right)=\operatorname{Var}\left(M_{1}\right)=\frac{7 n^{5}-20 n^{4}+75 n^{3}-40 n^{2}-322 n+300}{60 n^{4}} .
$$


Proof. The theorem can be seen to be true for $2 \leq n \leq 5$ using Table 1.1. Assume $n \geq 6$. Let $I_{n, 1}=\{(i, j, k): 1 \leq k<i<j \leq n\}, I_{n, 2}=\{(i, j, k): 1 \leq i<k<j \leq n\}$, and $I_{n}=I_{n, 1} \cup I_{n, 2}$. For $(i, j, k) \in I_{n}$ and $T \in \mathscr{T}_{n}$, let

$$
X_{(i, j, k)}(T)= \begin{cases}1, & i j k \text { is a local maximum in } T \text { rooted at } 1 \\ 0, & \text { otherwise; }\end{cases}
$$

hence,

$$
M_{1}=\sum_{(i, j, k) \in I_{n}} X_{(i, j, k)}
$$

We remind the reader that $i j, j k$ are always edges in a tree by using thick lines in our diagrams.

Expectation of $M_{1}$. We consider the following two cases according to the path $S$ of $T$ from 1 through $i j k$. Only $E\left(X_{(i, j, k)}\right) \neq 0$ need to be considered.

Case $1(i \neq 1)$. Here

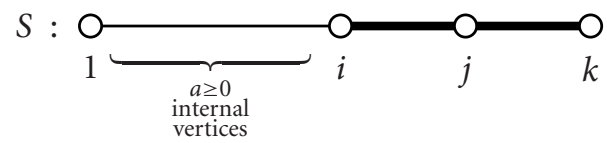

There are $(a+4) n^{n-a-5}$ trees in $\mathscr{T}_{n}$ containing a specific tree $S$ by Theorem 2.4 ; there are $(n-4)_{a}$ specific trees containing $a$ vertices between $1, i$; and there are $2\left(\begin{array}{c}n-1 \\ 3\end{array}\right)$ choices for $(i, j, k)$. Hence,

$$
\sum_{\substack{(i, j, k) \in I_{n} \\ 1 \neq i}} E\left(X_{(i, j, k)}\right)=\frac{(n-1)_{3}}{3 n^{3}} \sum_{a=0}^{n-4}(n-4)_{a} \frac{a+4}{n^{a}}=\frac{(n-1)_{3}}{3 n^{2}}
$$

by Lemma 2.2 .

Case $2(i=1)$. Here

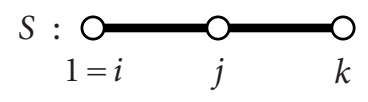

There are $3 n^{n-4}$ trees in $\mathscr{T}_{n}$ containing a specific tree $S$ by Theorem 2.4; and there are $\left(\begin{array}{c}n-1 \\ 2\end{array}\right)$ choices for $(j, k)$. Hence,

$$
\sum_{(1, j, k) \in I_{n}} E\left(X_{(i, j, k)}\right)=\frac{3(n-1)_{2}}{2 n^{2}}
$$


From (2.18), (2.19),

$$
E\left(M_{1}\right)=\frac{(n-1)_{3}}{3 n^{2}}+\frac{3(n-1)_{2}}{2 n^{2}}=\frac{2 n^{3}-3 n^{2}-5 n+6}{6 n^{2}} .
$$

Variance of $M_{1}$. Here

$$
M_{1}^{2}=\left(\sum_{(i, j, k) \in I_{n}} X_{(i, j, k)}\right)^{2}=M_{1}+\sum_{\left(\left(i_{1}, j_{1}, k_{1}\right),\left(i_{2}, j_{2}, k_{2}\right)\right) \in I_{n}^{*}} X_{\left(i_{1}, j_{1}, k_{1}\right)} X_{\left(i_{2}, j_{2}, k_{2}\right)},
$$

where $I_{n}^{*}=\left\{\left(\left(i_{1}, j_{1}, k_{1}\right),\left(i_{2}, j_{2}, k_{2}\right)\right) \in I_{n} \times I_{n}:\left(i_{1}, j_{1}, k_{1}\right) \neq\left(i_{2}, j_{2}, k_{2}\right)\right\}$.

First, we describe how we calculate $E\left(M_{1}^{2}\right)-E\left(M_{1}\right)$.

We first consider $3 \cdot 2=6$ cases according to $\#\left\{i_{1}, j_{1}, k_{1}, i_{2}, j_{2}, k_{2}\right\}=6,5$, or 4 , and, whether $1 \notin\left\{i_{1}, j_{1}, k_{1}, i_{2}, j_{2}, k_{2}\right\}$ or $1 \in\left\{i_{1}, j_{1}, k_{1}, i_{2}, j_{2}, k_{2}\right\}$. In each of these six cases, we further partition as described below.

For $\left(\left(i_{1}, j_{1}, k_{1}\right),\left(i_{2}, j_{2}, k_{2}\right)\right) \in I_{n}^{*}$, we consider the possible subtrees $S=S_{\left(\left(i_{1}, j_{1}, k_{1}\right),\left(i_{2}, j_{2}, k_{2}\right)\right)}$ of $[n]$ determined by the path from 1 to the second coordinate $i_{2} j_{2} k_{2}$ relative to the path from 1 to the first coordinate $i_{1} j_{1} k_{1}$. The possible subtrees $S^{\prime}=S_{\left(\left(i_{2}, j_{2}, k_{2}\right),\left(i_{1}, j_{1}, k_{1}\right)\right)}^{\prime}$ are included above by definition. Only $E\left(X_{\left(i_{1}, j_{1}, k_{1}\right)} X_{\left(i_{2}, j_{2}, k_{2}\right)}\right) \neq 0$ need to be considered. This gives nine types of subtrees of $[n]$ total among these six cases.

For the symmetric types $1,3,5,7$, and $9, S$ "looks like" $S^{\prime}$. We count the number $t_{S}$ of trees $T \in \mathscr{T}_{n}$ containing $S=S_{\left(\left(i_{1}, j_{1}, k_{1}\right),\left(i_{2}, j_{2}, k_{2}\right)\right)}$ and the number $i_{S}$ of such $\left(\left(i_{1}, j_{1}, k_{1}\right)\right.$, $\left.\left(i_{2}, j_{2}, k_{2}\right)\right)$. The product $i_{S} t_{S}$ counts each tree $T \in \mathscr{T}_{n}$ containing $S$ twice; once for $S$ and once for $S^{\prime}$. For each such tree $T, X_{\left(i_{1}, j_{1}, k_{1}\right)}(T) X_{\left(i_{2}, j_{2}, k_{2}\right)}(T)=1=X_{\left(i_{2}, j_{2}, k_{2}\right)}(T) X_{\left(i_{1}, j_{1}, k_{1}\right)}(T)$.

For the asymmetric types 2, 4, 6, and $8, S$ "looks different" than $S^{\prime}$. We introduce subtypes $S_{\left(\left(i_{1}, j_{1}, k_{1}\right),\left(i_{2}, j_{2}, k_{2}\right)\right)}^{x}$ and $S_{\left(\left(i_{1}, j_{1}, k_{1}\right),\left(i_{2}, j_{2}, k_{2}\right)\right)}^{y}$ so that $T \in \mathscr{T}_{n}$ contains $S^{x}=S_{\left(\left(i_{1}, j_{1}, k_{1}\right),\left(i_{2}, j_{2}, k_{2}\right)\right)}^{x}$ if and only if $T$ contains $S^{y}=S_{\left(\left(i_{2}, j_{2}, k_{2}\right),\left(i_{1}, j_{1}, k_{1}\right)\right)}^{y}$; note the different orders. We count the number $t_{S^{z}}$ of trees $T \in \mathscr{T}_{n}$ containing $S^{z}$ and the number $i_{S^{z}}$ of such $\left(\left(i_{1}, j_{1}, k_{1}\right),\left(i_{2}, j_{2}, k_{2}\right)\right)$ for $z=x, y$. The sum $i_{S^{x}} t_{S^{x}}+i_{S^{y}} t_{S^{y}}$ counts each tree $T \in \mathscr{T}_{n}$ containing $S^{x}$ and $S^{y}$; once for $S^{x}$ and once for $S^{y}$. For each such tree $T, X_{\left(i_{1}, j_{1}, k_{1}\right)}(T) X_{\left(i_{2}, j_{2}, k_{2}\right)}(T)=1=X_{\left(i_{2}, j_{2}, k_{2}\right)}(T) X_{\left(i_{1}, j_{1}, k_{1}\right)}(T)$.

For each type, the above count(s) are divided by $n^{n-2}$ then simplified using Lemma 2.2 and Corollary 2.3. Summing over the types of a particular case $i(1 \leq i \leq 6)$ gives

$$
\sum_{\substack{\left(\left(i_{1}, j_{1}, k_{1}\right),\left(i_{2}, j_{2}, k_{2}\right)\right) \in I_{n}^{*} \\ \text { case } i}} E\left(X_{\left(i_{1}, j_{1}, k_{1}\right)} X_{\left(i_{2}, j_{2}, k_{2}\right)}\right)
$$

The sum over all six cases is then $E\left(M_{1}^{2}\right)-E\left(M_{1}\right)$.

In what follows, $(n-1)_{6}=E_{n-7}(n)=0$ for $n=6$ and $E_{n-9}(n)=0$ for $n=6,7,8$ as usual. All cases appear for $n \geq 9$. 
Case $3\left(\#\left\{i_{1}, j_{1}, k_{1}, i_{2}, j_{2}, k_{2}\right\}=6,1 \notin\left\{i_{1}, j_{1}, k_{1}, i_{2}, j_{2}, k_{2}\right\}\right)$.

Type 1. Here

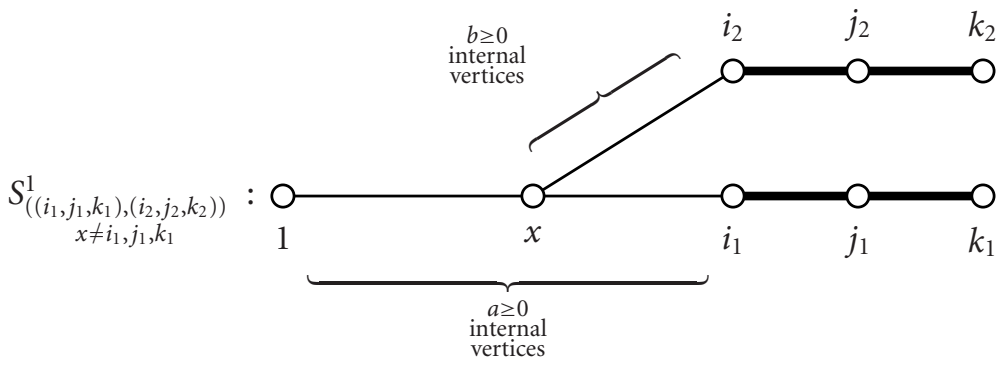

There are $(a+b+7) n^{n-a-b-8}$ trees in $\mathscr{T}_{n}$ containing a specific tree $S^{1}$ by Theorem 2.4; there are $(n-7)_{a+b}$ specific trees containing $a$ vertices between $1, i_{1}$ and $b$ vertices between $x, i_{2}$; there are $a+1$ choices for $x$; and there are $2\left(\begin{array}{c}n-1 \\ 3\end{array}\right) \cdot 2\left(\begin{array}{c}n-4 \\ 3\end{array}\right)$ such pairs $\left(\left(i_{1}, j_{1}, k_{1}\right)\right.$, $\left.\left(i_{2}, j_{2}, k_{2}\right)\right)$. Observe that $T$ contains $S_{\left(\left(i_{1}, j_{1}, k_{1}\right),\left(i_{2}, j_{2}, k_{2}\right)\right)}^{1}$ for $a, b, x$ if and only if $T$ contains $S_{\left(\left(i_{2}, j_{2}, k_{2}\right),\left(i_{1}, j_{1}, k_{1}\right)\right)}^{1}$ for $a^{\prime}, b^{\prime}, x$. Hence, (each such pair appears once)

$$
\begin{aligned}
\sum_{\substack{\left(\left(i_{1}, j_{1}, k_{1}\right),\left(i_{2}, j_{2}, k_{2}\right)\right) \in I_{n}^{*} \\
T \text { Type } 1}} E\left(X_{\left(i_{1}, j_{1}, k_{1}\right)} X_{\left(i_{2}, j_{2}, k_{2}\right)}\right) & =\frac{(n-1)_{6}}{9 n^{6}} \sum_{\substack{(a, b) \in \mathbb{N}^{2} \\
0 \leq a+b \leq n-7}}(n-7)_{a+b} \frac{(a+1)(a+b+7)}{n^{a+b}} \\
& =\frac{(n-1)_{6}}{9 n^{6}} \sum_{a=0}^{n-7}(n-7)_{a} \frac{a+1}{n^{a}} \sum_{b=0}^{n-a-7}(n-a-7)_{b} \frac{a+b+7}{n^{b}} \\
& =\frac{(n-1)_{6}}{9 n^{5}} \sum_{a=0}^{n-7}(n-7)_{a} \frac{a+1}{n^{a}} \\
& =\frac{(n-1)_{6}}{9 n^{5}}\left\{n-\frac{6(n-7) !}{n^{n-7}} E_{n-7}(n)\right\} \\
& =\frac{(n-1)_{6}}{9 n^{4}}-\frac{2(n-1) !}{3 n^{n-2}} E_{n-7}(n)
\end{aligned}
$$

by Lemma 2.2, and Corollary 2.3.

Type 2. First subtypes $2_{1}, 2_{2}, 2_{3}$ are

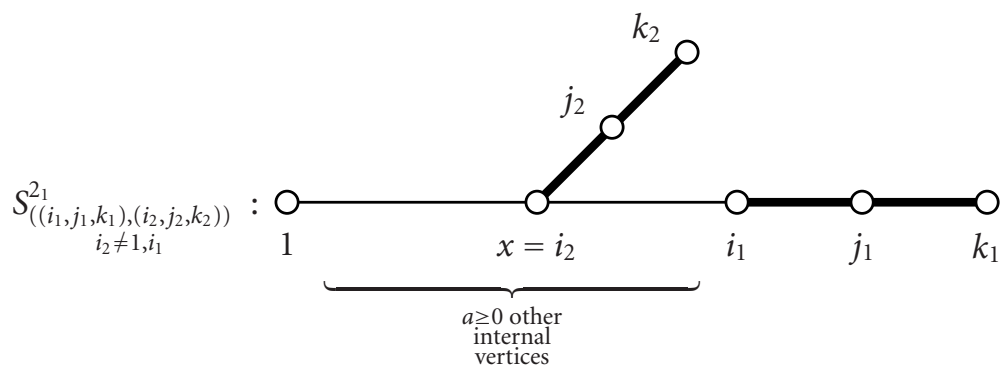




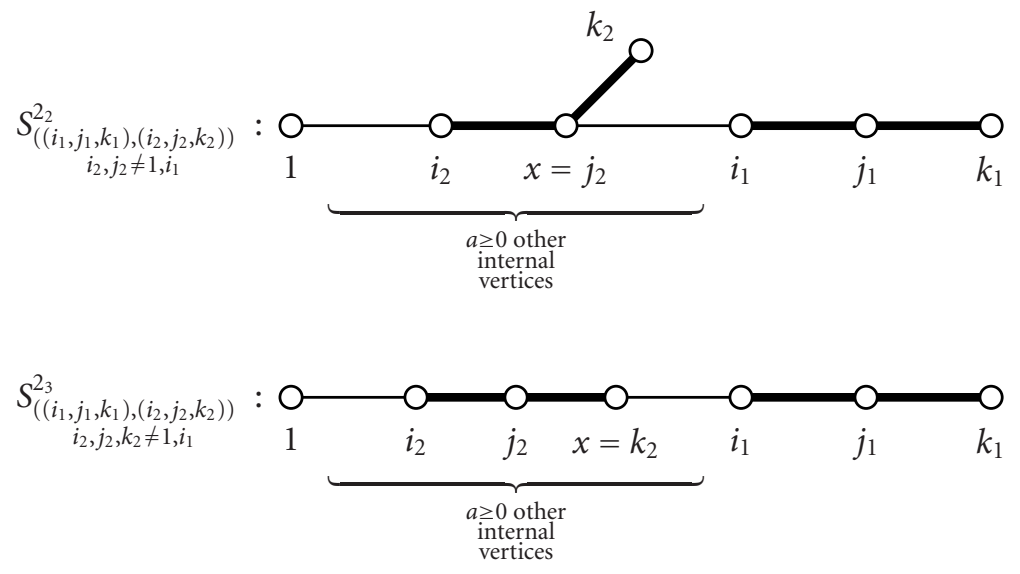

In each of the subcases, we have replaced one of the $a+1$ edges $u v$ between $1, i_{1}$, with the path $u x=i_{2} v, u i_{2} x=j_{2} v$ or $u i_{2} j_{2} x=k_{2} v$, where the rest of the path $i_{2} j_{2} k_{2}$ is as indicated. In each of these three subcases, there are $(a+7) n^{n-a-8}$ trees in $\mathscr{T}_{n}$ containing a specific tree $S^{2_{1}}, S^{2_{2}}, S^{2_{3}}$ by Theorem 2.4; there are $(n-7)_{a}$ specific trees containing $a$ other vertices between $1, i_{1}$; there are $a+1$ choices for $x$, equivalently, $u v$; and there are $2\left(\begin{array}{c}n-1 \\ 3\end{array}\right) \cdot 2\left(\begin{array}{c}n-4 \\ 3\end{array}\right)$ such pairs $\left(\left(i_{1}, j_{1}, k_{1}\right),\left(i_{2}, j_{2}, k_{2}\right)\right)$. Next, subtype $2_{4}$ is

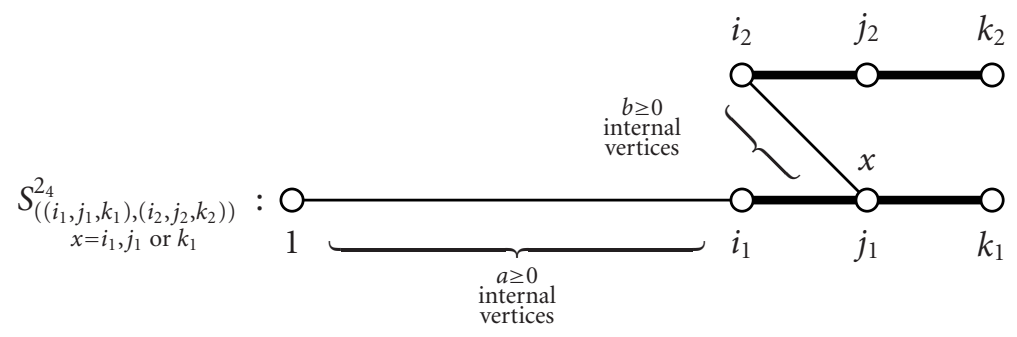

There are $(a+b+7) n^{n-a-b-8}$ trees in $\mathscr{T}_{n}$ containing a specific tree $S^{2_{4}}$ by Theorem 2.4; there are $(n-7)_{a+b}$ specific trees containing $a$ vertices between $1, i_{1}$ and $b$ vertices between $x, i_{2}$; there are 3 choices for $x=i_{1}, j_{1}$, or $k_{1}$; and there are $2\left(\begin{array}{c}n-1 \\ 3\end{array}\right) \cdot 2\left(\begin{array}{c}n-4 \\ 3\end{array}\right)$ such pairs $\left(\left(i_{1}, j_{1}, k_{1}\right),\left(i_{2}, j_{2}, k_{2}\right)\right)$. Observe that $T$ contains $S_{\left(\left(i_{1}, j_{1}, k_{1}\right),\left(i_{2}, j_{2}, k_{2}\right)\right)}^{2_{1,2} 3}$ for $a, x$ if and only if $T$ contains $S_{\left(\left(i_{2}, j_{2}, k_{2}\right),\left(i_{1}, j_{1}, k_{1}\right)\right)}^{2_{4}}$ for $a^{\prime}, b^{\prime}, x$. Hence, (each such pair appears once)

$$
\begin{aligned}
& \sum_{\substack{\left(\left(i_{1}, j_{1}, k_{1}\right),\left(i_{2}, j_{2}, k_{2}\right)\right) \in I_{n}^{*} \\
T \text { Type 2 }}} E\left(X_{\left(i_{1}, j_{1}, k_{1}\right)} X_{\left(i_{2}, j_{2}, k_{2}\right)}\right) \\
& =\frac{(n-1)_{6}}{9 n^{6}}\left\{3 \sum_{a=0}^{n-7}(n-7)_{a} \frac{(a+1)(a+7)}{n^{a}}+3 \sum_{\substack{(a, b) \in \mathbb{N}^{2} \\
0 \leq a+b \leq n-7}}(n-7)_{a+b} \frac{a+b+7}{n^{a+b}}\right\}
\end{aligned}
$$




$$
\begin{aligned}
& =\frac{(n-1)_{6}}{3 n^{6}}\left\{\sum_{a=0}^{n-7}(n-7)_{a} \frac{(a+1)(a+7)}{n^{a}}+\sum_{a=0}^{n-7} \frac{(n-7)_{a}}{n^{a}} \sum_{b=0}^{n-a-7}(n-a-7)_{b} \frac{a+b+7}{n^{b}}\right\} \\
& =\frac{(n-1)_{6}}{3 n^{6}}\left\{\frac{(n-7) !}{n^{n-8}}(n-5) E_{n-7}(n)-\frac{(n-7) !}{n^{n-8}}(2 n-7) E_{n-8}(n)+\frac{(n-7) !}{n^{n-9}} E_{n-9}(n)\right\} \\
& =\frac{(n-1)_{6}}{3 n^{6}}\left\{\frac{2(n-7) !}{n^{n-8}} E_{n-9}(n)+4 n-14\right\} \\
& =\frac{2(n-1) !}{3 n^{n-2}} E_{n-9}(n)+(4 n-14) \frac{(n-1)_{6}}{3 n^{6}}
\end{aligned}
$$

by Lemma 2.2 and Corollary 2.3. (The first 3 above is number of subcases and the second 3 is the number of choices for $x$.)

Summing (2.23), (2.24) gives the following equation:

$$
\sum_{\left(\left(i_{1}, j_{1}, k_{1}\right),\left(i_{2}, j_{2}, k_{2}\right)\right) \in I_{n}^{*}} E\left(X_{\left(i_{1}, j_{1}, k_{1}\right)} X_{\left(i_{2}, j_{2}, k_{2}\right)}\right)
$$

$\#\left\{i_{1}, j_{1}, k_{1}, i_{2}, j_{2}, k_{2}\right\}=6,1 \notin\left\{i_{1}, j_{1}, k_{1}, i_{2}, j_{2}, k_{2}\right\}$

$$
\begin{aligned}
& =\frac{(n-1)_{6}}{9 n^{4}}-\frac{2(n-1) !}{3 n^{n-2}} E_{n-7}(n)+\frac{2(n-1) !}{3 n^{n-2}} E_{n-9}(n)+(4 n-14) \frac{(n-1)_{6}}{3 n^{6}} \\
& =\frac{(n-1)_{6}}{9 n^{4}}-\frac{2}{3}\left\{\frac{(n-1)_{6}}{n^{5}}+\frac{(n-1)_{7}}{n^{6}}-(2 n-7) \frac{(n-1)_{6}}{n^{6}}\right\} \\
& =\frac{(n-1)_{6}}{9 n^{4}} .
\end{aligned}
$$

Case $4\left(\#\left\{i_{1}, j_{1}, k_{1}, i_{2}, j_{2}, k_{2}\right\}=5,1 \notin\left\{i_{1}, j_{1}, k_{1}, i_{2}, j_{2}, k_{2}\right\}\right)$.

Type 3. Here

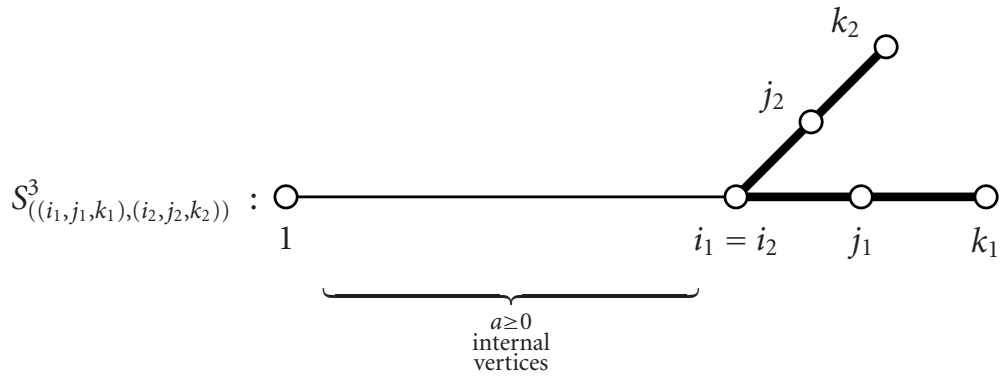

There are $(a+6) n^{n-a-7}$ trees in $\mathscr{T}_{n}$ containing a specific tree $S^{3}$ by Theorem 2.4 ; there are $(n-6)_{a}$ specific trees containing $a$ vertices between $1, i_{1}$; and there are $16\left(\begin{array}{c}n-1 \\ 5\end{array}\right)$ such pairs $\left(\left(i_{1}, j_{1}, k_{1}\right),\left(i_{2}, j_{2}, k_{2}\right)\right)$ (for 5 elements in $\{2, \ldots, n\}$, there are $2 \cdot 6=12$ pairs with largest 
elements $j_{1}, j_{2}$, and there are $2 \cdot 2=4$ pairs with $j_{1}>k_{1}>j_{2}$ or $\left.j_{2}>k_{2}>j_{1}\right)$. Observe that $T$ contains $S_{\left(\left(i_{1}, j_{1}, k_{1}\right),\left(i_{2}, j_{2}, k_{2}\right)\right)}^{3}$ if and only if $T$ contains $S_{\left(\left(i_{2}, j_{2}, k_{2}\right),\left(i_{1}, j_{1}, k_{1}\right)\right)}^{3}$. Hence, (each such pair appears once)

$$
\sum_{\substack{\left.\left(i_{1}, j_{1}, k_{1}\right),\left(i_{2}, j_{2}, k_{2}\right)\right) \in I_{n}^{*} \\ T \text { Type 3 }}} E\left(X_{\left(i_{1}, j_{1}, k_{1}\right)} X_{\left(i_{2}, j_{2}, k_{2}\right)}\right)=\frac{2(n-1)_{5}}{15 n^{5}} \sum_{a=0}^{n-6}(n-6)_{a} \frac{a+6}{n^{a}}=\frac{2(n-1)_{5}}{15 n^{4}}
$$

by Corollary 2.3.

Type 4. First subtypes $4_{1}, 4_{2}$ are

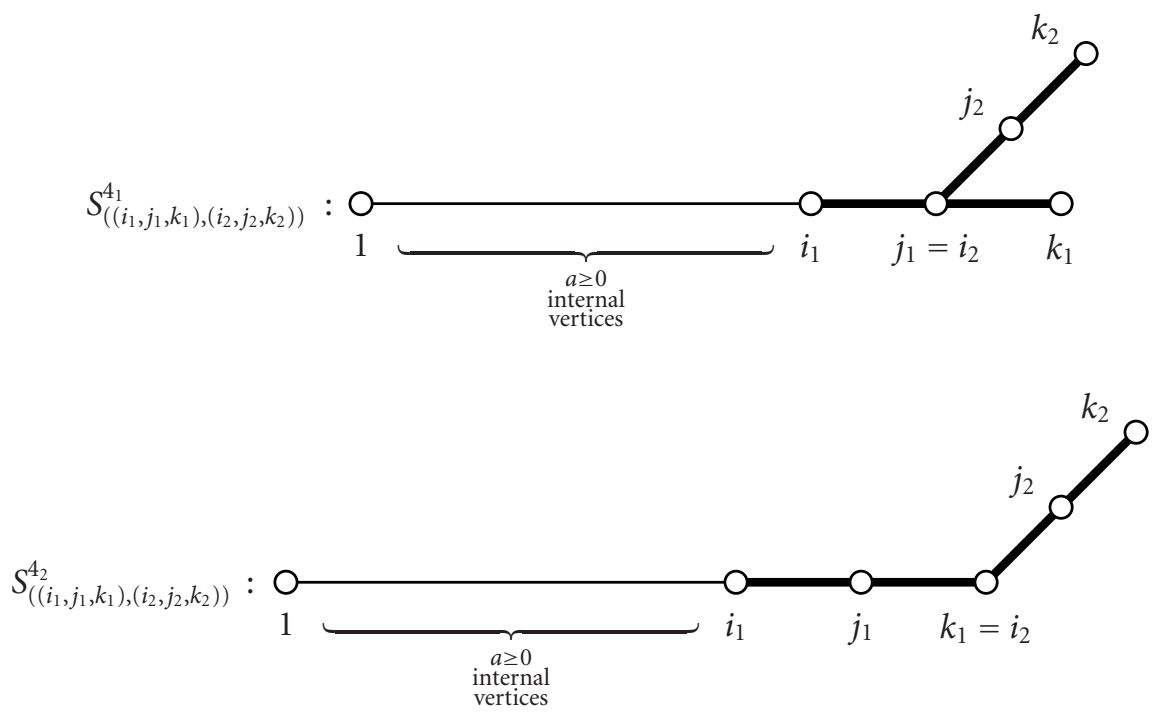

In either subcase, there are $(a+6) n^{n-a-7}$ trees in $\mathscr{T}_{n}$ containing a specific tree $S^{4_{1}}, S^{4_{2}}$ by Theorem 2.4; there are $(n-6)_{a}$ specific trees containing $a$ vertices between $1, i_{1}$; there are $24\left(\begin{array}{c}n-1 \\ 5\end{array}\right)$ such pairs $\left(\left(i_{1}, j_{1}, k_{1}\right),\left(i_{2}, j_{2}, k_{2}\right)\right)$ total (for 5 elements in $\{2, \ldots, n\}$; there are $6+2=8$ pairs with $j_{2}>i_{2}=j_{1}$ or $j_{2}>k_{2}>i_{2}=j_{1}$ for $4_{1}$; there are $2 \cdot 6=12$ pairs with largest elements $j_{1}, j_{2}$; and there are $2 \cdot 2=4$ pairs with $j_{1}>i_{1}>j_{2}$ or $j_{2}>k_{2}>j_{1}$ for $4_{2}$ ). Next subtypes $4_{3}, 4_{4}$ are

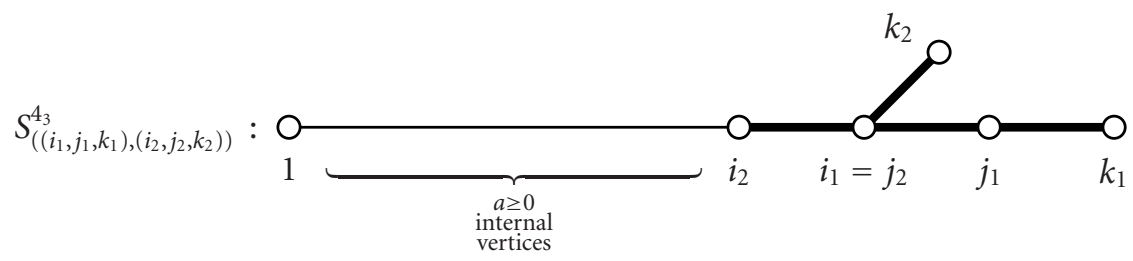




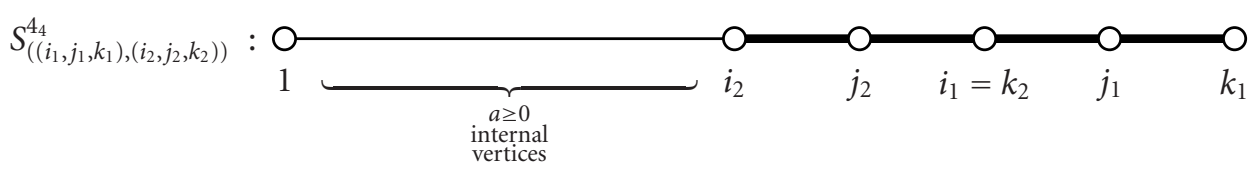

In either subcase, there are $(a+6) n^{n-a-7}$ trees in $\mathscr{T}_{n}$ containing a specific tree $S^{4_{3}}, S^{4_{4}}$ by Theorem 2.4; there are $(n-6)_{a}$ specific trees containing $a$ vertices between $1, i_{2}$; and there are $24\left(\begin{array}{c}n-1 \\ 5\end{array}\right)$ such pairs $\left(\left(i_{1}, j_{1}, k_{1}\right),\left(i_{2}, j_{2}, k_{2}\right)\right)$ total (for 5 elements in $\{2, \ldots, n\}$, there are $6+2=8$ pairs with $j_{1}>i_{1}=j_{2}$ or $j_{1}>k_{1}>i_{1}=j_{2}$ for $4_{3}$; there are $2 \cdot 6=12$ pairs with largest elements $j_{1}, j_{2}$, and there are $2 \cdot 2=4$ pairs with $j_{2}>i_{2}>j_{1}$ or $j_{1}>k_{1}>j_{2}$ for $\left.4_{4}\right)$. Observe that $T$ contains $S_{\left(\left(i_{1}, j_{1}, k_{1}\right),\left(i_{2}, j_{2}, k_{2}\right)\right)}^{4_{i}}$ if and only if $T$ contains $S_{\left(\left(i_{2}, j_{2}, k_{2}\right),\left(i_{1}, j_{1}, k_{1}\right)\right)}^{4_{i+2}}$ for $i=1,2$. Hence, (each such pair appears once)

$$
\sum_{\substack{\left(\left(i_{1}, j_{1}, k_{1}\right),\left(i_{2}, j_{2}, k_{2}\right)\right) \in I_{n}^{*} \\ T \text { Type } 4}} E\left(X_{\left(i_{1}, j_{1}, k_{1}\right)} X_{\left(i_{2}, j_{2}, k_{2}\right)}\right)=\frac{2(n-1)_{5}}{5 n^{5}} \sum_{a=0}^{n-6}(n-6)_{a} \frac{a+6}{n^{a}}=\frac{2(n-1)_{5}}{5 n^{4}}
$$

by Corollary 2.3. (The number of subcases has been accounted for.) Summing (2.26), (2.27) gives the following equation:

$$
\sum_{\substack{\left(\left(i_{1}, j_{1}, k_{1}\right),\left(i_{2}, j_{2}, k_{2}\right)\right) \in I_{n}^{*} \\ \#\left\{i_{1}, j_{1}, k_{1}, i_{2}, j_{2}, k_{2}\right\}=5,1 \notin\left\{i_{1}, j_{1}, k_{1}, i_{2}, j_{2}, k_{2}\right\}}} E\left(X_{\left(i_{1}, j_{1}, k_{1}\right)} X_{\left(i_{2}, j_{2}, k_{2}\right)}\right)=\frac{2(n-1)_{5}}{15 n^{4}}+\frac{2(n-1)_{5}}{5 n^{4}}=\frac{8(n-1)_{5}}{15 n^{4}} .
$$

Case $5\left(\#\left\{i_{1}, j_{1}, k_{1}, i_{2}, j_{2}, k_{2}\right\}=4,1 \notin\left\{i_{1}, j_{1}, k_{1}, i_{2}, j_{2}, k_{2}\right\}\right)$.

Type 5. Here

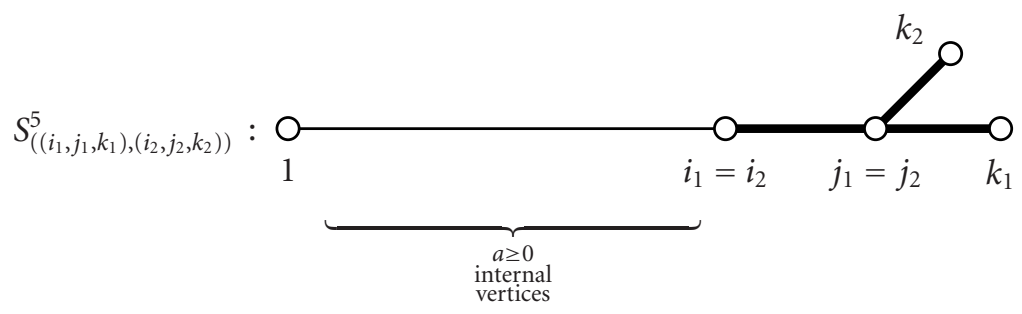

There are $(a+5) n^{n-a-6}$ trees in $\mathscr{T}_{n}$ containing a specific tree $S^{5}$ by Theorem 2.4 ; there are $(n-5)_{a}$ specific trees containing $a$ vertices between $1, i_{1}$; and there are $6\left(\begin{array}{c}n-1 \\ 4\end{array}\right)$ such pairs 
$\left(\left(i_{1}, j_{1}, k_{1}\right),\left(i_{2}, j_{2}, k_{2}\right)\right)$. Observe that $T$ contains $S_{\left(\left(i_{1}, j_{1}, k_{1}\right),\left(i_{2}, j_{2}, k_{2}\right)\right)}^{5}$ if and only if $T$ contains $S_{\left(\left(i_{2}, j_{2}, k_{2}\right),\left(i_{1}, j_{1}, k_{1}\right)\right)}^{5}$. Hence, (each such pair appears once)

$$
\sum_{\substack{\left(\left(i_{1}, j_{1}, k_{1}\right),\left(i_{2}, j_{2}, k_{2}\right)\right) \in \in_{n}^{*} \\ \#\left\{i_{1}, j_{1}, k_{1}, i_{2}, j_{2}, k_{2}\right\}=4,1 \notin\left\{i_{1}, j_{1}, k_{1}, i_{2}, j_{2}, k_{2}\right\}}} E\left(X_{\left(i_{1}, j_{1}, k_{1}\right)} X_{\left(i_{2}, j_{2}, k_{2}\right)}\right)=\frac{(n-1)_{4}}{4 n^{4}} \sum_{a=0}^{n-5}(n-5)_{a} \frac{a+5}{n^{a}}=\frac{(n-1)_{4}}{4 n^{3}}
$$

by Corollary 2.3.

Case $6\left(\#\left\{i_{1}, j_{1}, k_{1}, i_{2}, j_{2}, k_{2}\right\}=6,1 \in\left\{i_{1}, j_{1}, k_{1}, i_{2}, j_{2}, k_{3}\right\}\right)$.

Type 6. First subtypes $6_{1}, 6_{2}, 6_{3}$ are
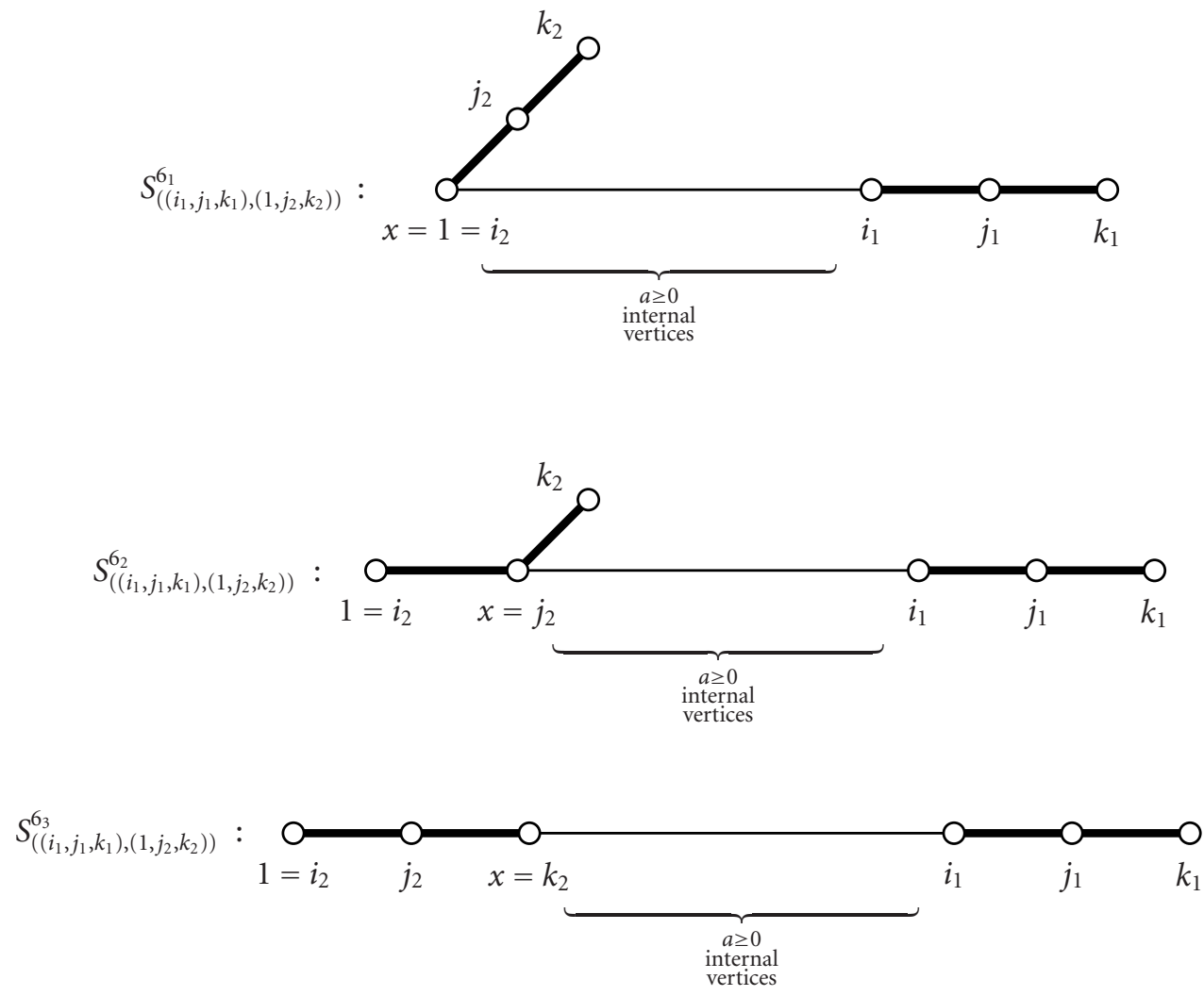

In each of these three subcases, there are $(a+6) n^{n-a-7}$ trees in $\mathscr{T}_{n}$ containing a specific tree $S^{6_{1}}, S^{6_{2}}, S^{6_{3}}$ by Theorem 2.4; there are $(n-6)_{a}$ specific trees containing $a$ vertices between $x, i_{1}$; and there are $\left(\begin{array}{c}n-1 \\ 2\end{array}\right) \cdot 2\left(\begin{array}{c}n-3 \\ 3\end{array}\right)$ such pairs $\left(\left(i_{1}, j_{1}, k_{1}\right),\left(1, i_{2}, j_{2}\right)\right)$. Next 
subtype $6_{4}$ is

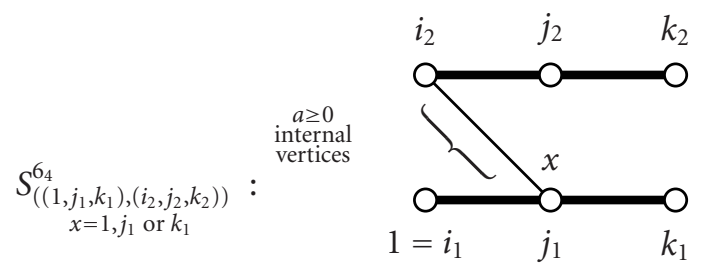

There are $(a+6) n^{n-a-7}$ trees in $\mathscr{T}_{n}$ containing a specific tree $S^{6_{4}}$ by Theorem 2.4; there are $(n-6)_{a}$ specific trees containing $a$ vertices between $x, i_{2}$; there are 3 choices for $x=$ $1, j_{1}$, or $k_{1}$; and there are $\left(\begin{array}{c}n-1 \\ 2\end{array}\right) \cdot 2\left(\begin{array}{c}n-3 \\ 3\end{array}\right)$ such pairs $\left(\left(1, j_{1}, k_{1}\right),\left(i_{2}, j_{2}, k_{2}\right)\right)$. Observe that $T$ contains $S_{\left(\left(i_{1}, j_{1}, k_{1}\right),\left(1, j_{2}, k_{2}\right)\right)}^{6_{1,23}}$ for $a, x$ if and only if $T$ contains $S_{\left(\left(1, j_{2}, k_{2}\right),\left(i_{1}, j_{1}, k_{1}\right)\right)}^{6_{4}}$ for $a, x$. Hence, (each such pair appears once)

$$
\begin{gathered}
\sum_{\substack{\left(\left(i_{1}, j_{1}, k_{1}\right),\left(i_{2}, j_{2}, k_{2}\right)\right) \in I_{n}^{*} \\
\#\left\{i_{1}, j_{1}, k_{1}, i_{2}, j_{2}, k_{2}\right\}=6,1 \in\left\{i_{1}, j_{1}, k_{1}, i_{2}, j_{2}, k_{2}\right\}}} E\left(X_{\left(i_{1}, j_{1}, k_{1}\right)} X_{\left(i_{2}, j_{2}, k_{2}\right)}\right) \\
=\frac{(n-1)_{5}}{6 n^{5}}\left\{3 \sum_{a=0}^{n-6}(n-6) a \frac{a+6}{n^{a}}+3 \sum_{a=0}^{n-6}(n-6)_{a} \frac{a+6}{n^{a}}\right\} \\
=\frac{(n-1)_{5}}{n^{4}}
\end{gathered}
$$

by Corollary 2.3. (The first 3 and second 3 above are the number of subcases, i.e., choices for $x$.)

Case $7\left(\#\left\{i_{1}, j_{1}, k_{1}, i_{2}, j_{2}, k_{2}\right\}=5,1 \in\left\{i_{1}, j_{1}, k_{1}, i_{2}, j_{2}, k_{2}\right\}\right)$.

Type 7. Here

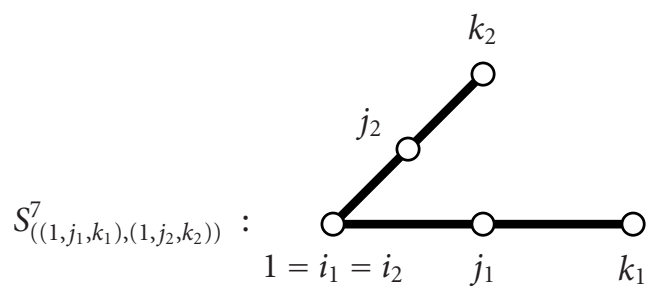

There are $5 n^{n-6}$ trees in $\mathscr{T}_{n}$ containing a specific tree $S^{7}$ by Theorem 2.4; and there are $\left(\begin{array}{c}n-1 \\ 2\end{array}\right) \cdot\left(\begin{array}{c}n-3 \\ 2\end{array}\right)$ such pairs $\left(\left(1, j_{1}, k_{1}\right),\left(1, j_{2}, k_{2}\right)\right)$. Observe that $T$ contains $S_{\left(\left(1, j_{1}, k_{1}\right),\left(1, j_{2}, k_{2}\right)\right)}^{7}$ if and only if $T$ contains $S_{\left(\left(1, j_{2}, k_{2}\right),\left(1, j_{1}, k_{1}\right)\right)}^{7}$. Hence, (each such pair occurs once)

$$
\sum_{\substack{\left(\left(1, j_{1}, k_{1}\right),\left(1, j_{2}, k_{2}\right)\right) \in I_{n}^{*} \\ T \text { Type } 7}} E\left(X_{\left(1, j_{1}, k_{1}\right)} X_{\left(1, j_{2}, k_{2}\right)}\right)=\frac{5(n-1)_{4}}{4 n^{4}} .
$$


Type 8. First subtypes $8_{1}, 8_{2}$ are
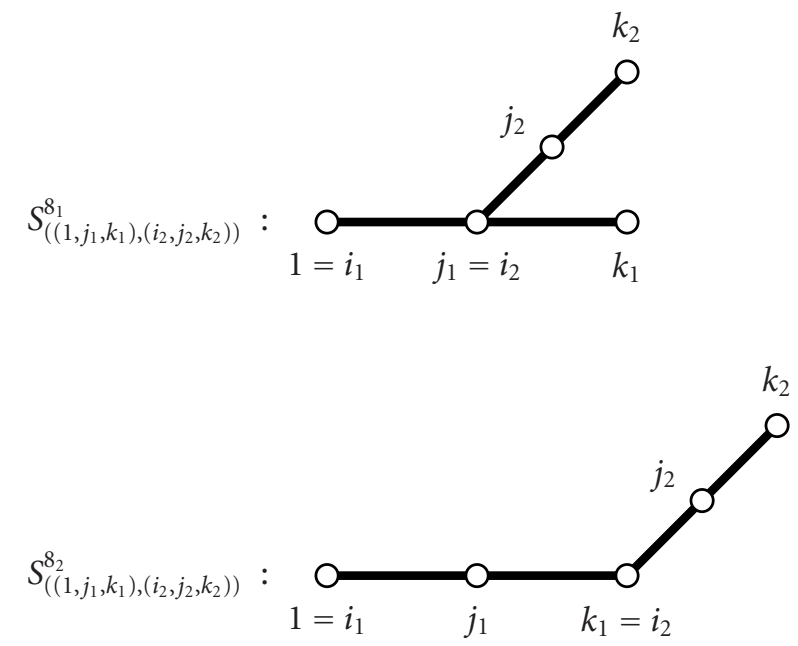

In either subcase, there are $5 n^{n-6}$ trees in $\mathscr{T}_{n}$ containing a specific tree $S^{8_{1}}, S^{8_{2}}$ by Theorem 2.4; and there are $8\left(\begin{array}{c}n-1 \\ 4\end{array}\right)$ such pairs $\left(\left(1, j_{1}, k_{1}\right),\left(i_{2}, j_{2}, k_{2}\right)\right)$ total (for 4 elements in $\{2, \ldots, n\}$, there are $2+1=3$ pairs with $j_{2}>j_{1}=i_{2}$ or $j_{2}>k_{2}>i_{2}=j_{1}$ for $8_{1}$; and there are $2+2+$ $1=5$ pairs with largest elements $j_{1}, j_{2}$ or $j_{2}>k_{2}>j_{1}$ for $8_{2}$ ). Next subtypes $8_{3}, 8_{4}$ are

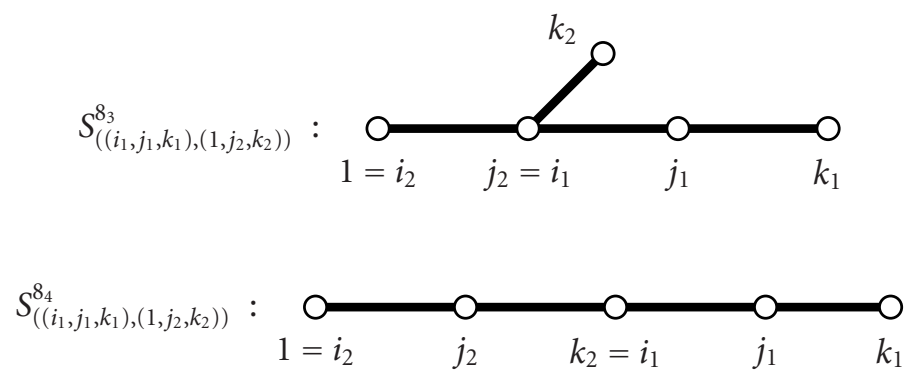

In either subcase, there are $5 n^{n-6}$ trees in $\mathscr{T}_{n}$ containing a specific tree $S^{8_{3}}, S^{8_{4}}$ by Theorem 2.4; and there are $8\left(\begin{array}{c}n-1 \\ 4\end{array}\right)$ such pairs $\left(\left(i_{1}, j_{1}, k_{1}\right),\left(1, j_{2}, k_{2}\right)\right)$ total (for 4 elements in $\{2, \ldots, n\}$, there are $2+1=3$ pairs with $j_{1}>j_{2}=i_{1}$ or $j_{1}>k_{1}>i_{1}=j_{2}$ for $8_{3}$; and there are $2+2+$ $1=5$ pairs with largest elements $j_{1}, j_{2}$ or $j_{1}>k_{1}>j_{2}$ for $\left.8_{4}\right)$. Observe that $T$ contains $S_{\left(\left(1, j_{1}, k_{1}\right),\left(i_{2}, j_{2}, k_{2}\right)\right)}^{8_{i}}$ if and only if $T$ contains $S_{\left(\left(i_{2}, j_{2}, k_{2}\right),\left(1, j_{1}, k_{1}\right)\right)}^{8_{i+2}}$ for $i=1,2$. Hence, (each such pair occurs once)

$$
\sum_{\substack{\left(\left(i_{1}, j_{1}, k_{1}\right),\left(i_{2}, j_{2}, k_{2}\right)\right) \in I_{n}^{*} \\ T \text { Type 8 }}} E\left(X_{\left(i_{1}, j_{1}, k_{1}\right)} X_{\left(i_{2}, j_{2}, k_{2}\right)}\right)=\frac{5(n-1)_{4}}{3 n^{4}}+\frac{5(n-1)_{4}}{3 n^{4}}=\frac{10(n-1)_{4}}{3 n^{4}} .
$$


(The number of subcases has been accounted for.) Summing (2.31), (2.32) gives the following equation:

$$
\begin{array}{r}
\sum_{\substack{\left(\left(i_{1}, j_{1}, k_{1}\right),\left(i_{2}, j_{2}, k_{2}\right)\right) \in I_{n}^{*} \\
\#\left\{i_{1}, j_{1}, k_{1}, i_{2}, j_{2}, k_{2}\right\}=5,1 \in\left\{i_{1}, j_{1}, k_{1}, i_{2}, j_{2}, k_{2}\right\}}} E\left(X_{\left(i_{1}, j_{1}, k_{1}\right)} X_{\left(i_{2}, j_{2}, k_{2}\right)}\right) \\
=\frac{5(n-1)_{4}}{4 n^{4}}+\frac{10(n-1)_{4}}{3 n^{4}}=\frac{55(n-1)_{4}}{12 n^{4}} .
\end{array}
$$

Case $8\left(\#\left\{i_{1}, j_{1}, k_{1}, i_{2}, j_{2}, k_{2}\right\}=4,1 \in\left\{i_{1}, j_{1}, k_{1}, i_{2}, j_{2}, k_{2}\right\}\right)$.

Type 9. Here

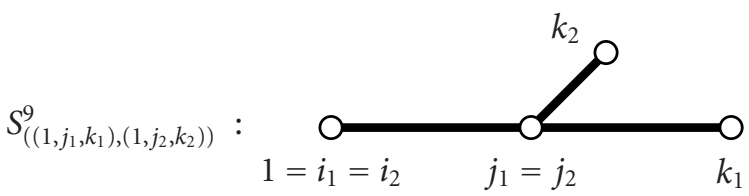

There are $4 n^{n-5}$ trees in $\mathscr{T}_{n}$ containing a specific tree $S^{9}$ by Theorem 2.4; and there are $2\left(\begin{array}{c}n-1 \\ 3\end{array}\right)$ such pairs $\left(\left(1, j_{1}, k_{1}\right),\left(1, j_{2}, k_{2}\right)\right)$. Observe that $T$ contains $S_{\left(\left(1, j_{1}, k_{1}\right),\left(1, j_{2}, k_{2}\right)\right)}^{9}$ if and only if $T$ contains $S_{\left(\left(1, j_{2}, k_{2}\right),\left(1, j_{1}, k_{1}\right)\right)}^{9}$. Hence, (each such pair occurs once)

$$
\sum_{\substack{\left(\left(i_{1}, j_{1}, k_{1}\right),\left(i_{2}, j_{2}, k_{2}\right)\right) \in I_{n}^{*} \\ \#\left\{i_{1}, j_{1}, k_{1}, i_{2}, j_{2}, k_{2}\right\}=4,1 \in\left\{i_{1}, j_{1}, k_{1}, i_{2}, j_{2}, k_{2}\right\}}} E\left(X_{\left(i_{1}, j_{1}, k_{1}\right)} X_{\left(i_{2}, j_{2}, k_{2}\right)}\right)=\frac{4(n-1)_{3}}{3 n^{3}} .
$$

After all this preparation, we are now able to find the second moment and the variance of $M_{1}$. From (2.21), summing (2.20), (2.25), (2.28)-(2.30), (2.33), (2.34) gives

$$
\begin{aligned}
E\left(M_{1}^{2}\right)= & \frac{2 n^{3}-3 n^{2}-5 n+6}{6 n^{2}}+\frac{(n-1)_{6}}{9 n^{4}}+\frac{8(n-1)_{5}}{15 n^{4}}+\frac{(n-1)_{4}}{4 n^{3}} \\
& +\frac{(n-1)_{5}}{n^{4}}+\frac{55(n-1)_{4}}{12 n^{4}}+\frac{4(n-1)_{3}}{3 n^{3}} \\
= & \frac{20 n^{6}-39 n^{5}-115 n^{4}+495 n^{3}-175 n^{2}-1266 n+1080}{180 n^{4}} .
\end{aligned}
$$

Hence, (2.20), (2.35) give

$$
\sigma^{2}\left(M_{1}\right)=\operatorname{Var}\left(M_{1}\right)=\frac{7 n^{5}-20 n^{4}+75 n^{3}-40 n^{2}-322 n+300}{60 n^{4}} .
$$

As a consequence of Theorem 2.5, a.a.s. on $\left\{\Omega_{n}\right\}, M_{1}(T)$ and $m_{n}(T)$ belong to a relatively small interval for $T \in \mathscr{T}_{n}$. Again, $M_{1}=M_{1, n}$.

Corollary 2.6. For $\left\{\Omega_{n}\right\}$,

$$
\operatorname{Pr}\left(\left|M_{1}-E\left(M_{1}\right)\right|<\omega(n) \sigma\left(M_{1}\right)\right) \longrightarrow 1 \quad \text { as } n \longrightarrow \infty,
$$


where $\omega(n) \rightarrow \infty$ arbitarily slowly as $n \rightarrow \infty$. Hence, a.a.s. on $\left\{\Omega_{n}\right\}$,

$$
\frac{n}{3}-\omega(n) n^{0.5}<M_{1}<\frac{n}{3}+\omega(n) n^{0.5}
$$

where $\omega(n) \rightarrow \infty$ arbitarily slowly as $n \rightarrow \infty$.

Proof. By Chebyshev's inequality,

$$
\operatorname{Pr}\left(\left|M_{1}-E\left(M_{1}\right)\right| \geq \omega(n) \sigma\left(M_{1}\right)\right) \leq \frac{1}{\omega^{2}(n)} \longrightarrow 0 \quad \text { as } n \longrightarrow \infty,
$$

provided that $\omega(n) \rightarrow \infty$ as $n \rightarrow \infty$. This implies our result.

\section{Acknowledgment}

I wish to thank both referees for comments and suggestions. The detailed report by one referee in particular led to this much improved version of the note.

\section{References}

[1] L. H. Clark, Ascents and descents in random trees, preprint, 2004.

[2] Inversions in random trees, preprint, 2004.

[3] R. Durrett, Probability. Theory and Examples, The Wadsworth \& Brooks/Cole Statistics/Probability Series, Wadsworth \& Brooks/Cole Advanced Books \& Software, California, 1991.

[4] Ö. Eğecioğlu and J. B. Remmel, Bijections for Cayley trees, spanning trees, and their q-analogues, J. Combin. Theory Ser. A 42 (1986), no. 1, 15-30.

[5] I. M. Gessel, Counting forests by descents and leaves, Electron. J. Combin. 3 (1996), no. 2, Research Paper 8, 1-5.

[6] I. M. Gessel, B. E. Sagan, and Y.-N. Yeh, Enumeration of trees by inversions, J. Graph Theory 19 (1995), no. 4, 435-459.

[7] C. L. Mallows and J. Riordan, The inversion enumerator for labeled trees, Bull. Amer. Math. Soc. 74 (1968), 92-94.

[8] J. W. Moon, Counting Labelled Trees, Canadian Mathematical Monographs, no. 1, Canadian Mathematical Congress, Quebec, 1970.

[9] - The expected number of inversions in a random tree, Proc. Louisiana Conf. on Combinatorics, Graph Theory and Computing (Louisiana State Univ., Baton Rouge, La, 1970), Louisiana State University, Louisiana, 1970, pp. 375-382.

[10] D. Warren and E. Seneta, Peaks and Eulerian numbers in a random sequence, J. Appl. Probab. 33 (1996), no. 1, 101-114.

Lane Clark: Department of Mathematics, College of Science, Southern Illinois University Carbondale, Carbondale, IL 62901-4408, USA

E-mail address: 1clark@math.siu.edu 


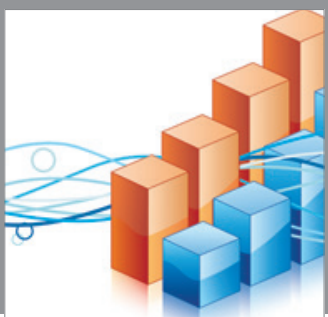

Advances in

Operations Research

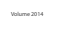

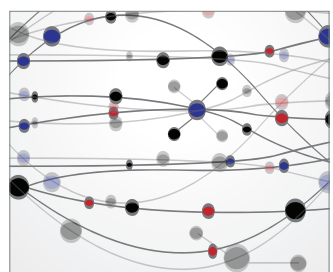

\section{The Scientific} World Journal
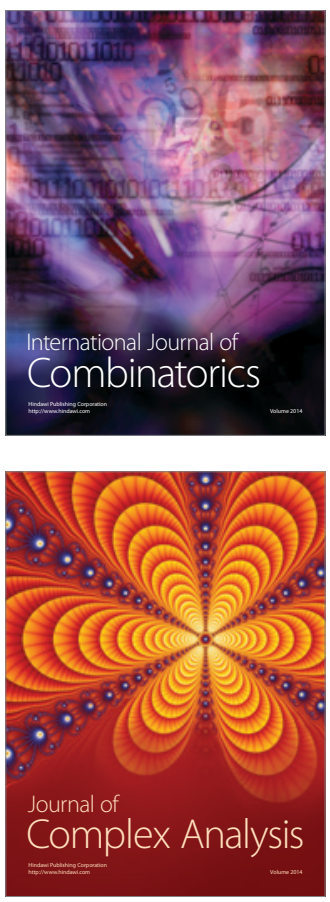

International Journal of

Mathematics and

Mathematical

Sciences
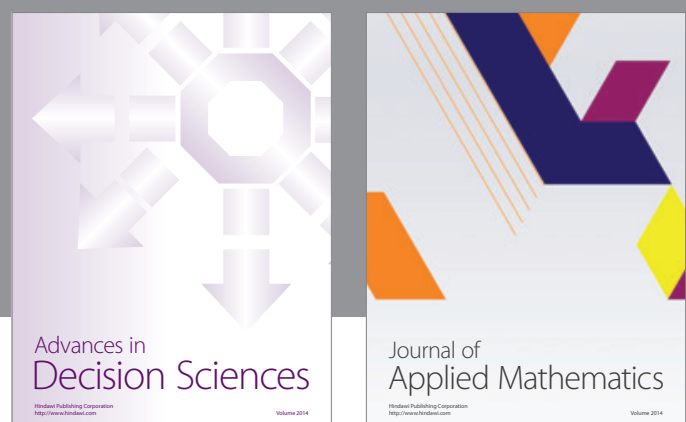

Journal of

Applied Mathematics
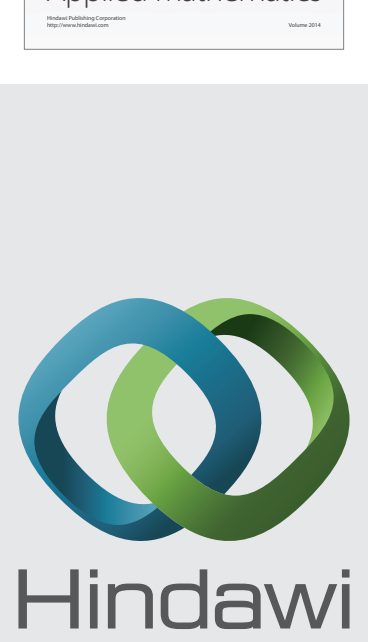

Submit your manuscripts at http://www.hindawi.com
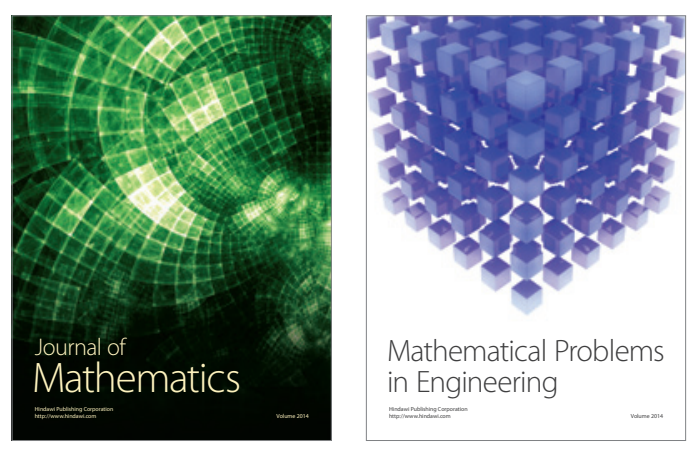

Mathematical Problems in Engineering
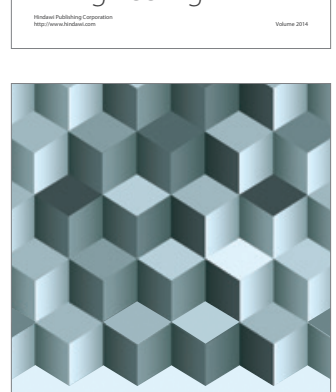

Journal of

Function Spaces
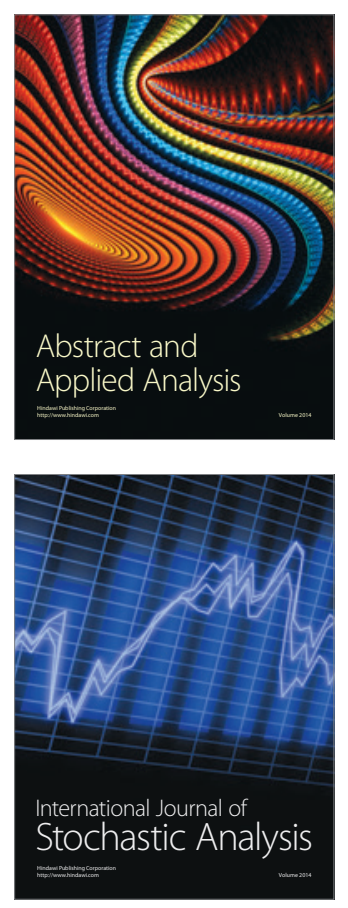

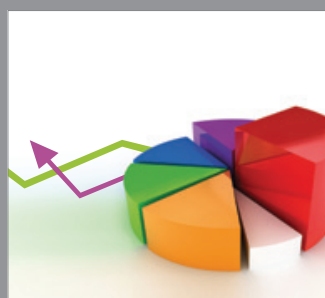

ournal of

Probability and Statistics

Promensencen
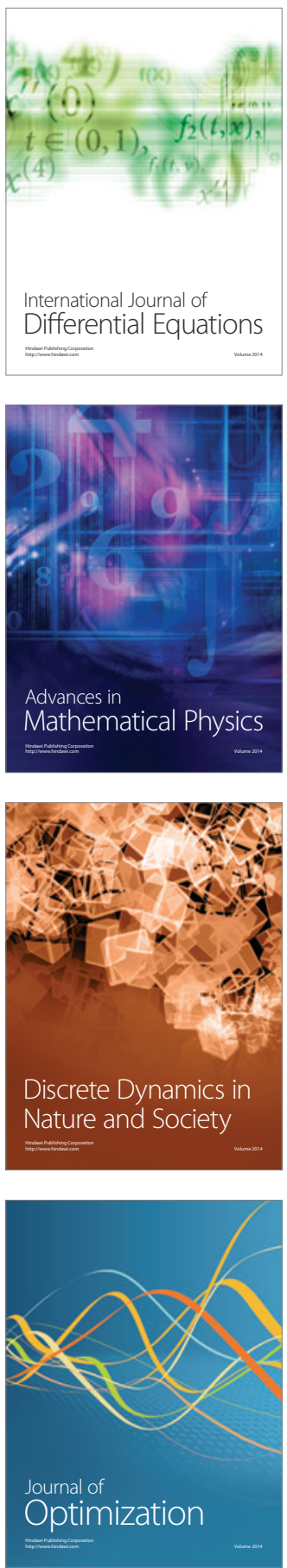